\title{
5-(Azulen-1-yldiazenyl)tetrazoles; Syntheses and Properties
}

\author{
LIVIU BIRZAN ${ }^{*}$, MIHAELA CRISTEA ${ }^{1}$, VICTORITA TECUCEANU ${ }^{1}$, \\ ANAMARIA HANGANU ${ }^{1}$, ELEONORA-MIHAELA UNGUREANU ${ }^{2}$, \\ ALEXANDRU C. RAZUS ${ }^{1}$ \\ ${ }^{1}$ Romanian Academy, Institute of Organic Chemistry "C. D. Nenitzescu”, 202B Spl. Independentei, 060023, Bucharest, \\ Romania \\ ${ }^{2}$ University Politehnica of Bucharest, 1-7 Gheorghe Polizu Str., 011061, Bucharest, Romania
}

\begin{abstract}
The tetrazole-5-diazonium salt, obtained by diazotizing 5-aminotetrazole in strong acid solution, was coupled with azulenes in the presence of pyridine to produce 5-(azulen-1yldiazenyl)tetrazoles in good yields. The tetrazole moiety was monoalkylated in 1-and in 2-position using dimethyl sulfate for methylation and benzyl bromide for benzylation. The excess of alkylating agent generated in small amount tetrazole dialkylated salts. The synthesized compounds were characterized and some of their properties have been investigated, such as optical, magnetical and electrical.
\end{abstract}

Keywords: azotetrazole, azulene, alkylation, $N M R$, $U v$-vis, redox potentials

\section{Introduction}

Since 1885 when Bladin synthesized 1-phenyl-5-cyanotetrazole [1], the first 5-membered heterocycle containing 4 nitrogen atoms, there has been a continuing interest in compounds containing this system in the molecule and a number of exhaustive reviews were published over time [2-5].

The fruitful utility in medicinal chemistry as biologically active compounds of tetrazole products, $[4,6,7]$ as well as its technical uses, have also stimulated the development of their synthesis. Much attention has been paid to the study of the physicochemical properties of tetrazoles, mainly for their remarkable reactivity observing many peculiarities of tetrazole system such as its charge distribution over its substituted derivatives that influence the reactions routes. The substitution of carbon or nitrogen atom in the heterocycle was thoroughly studied [8,9] as well as the possibility to build complexes [10,11] and salts with metals [12]. Another goal of researchers has been the study of diazenes containing the tetrazole system. The preparation of these compounds has begun since 1898, when Thiele synthesized azotetrazole and substituted tetrazole diazenes with 4-(dimethylamino)phenyl and $\beta$-naphthyl [13]. Salts of azotetrazole dianion with protonated nitrogen bases such as guanidinium are gas generators with the potential use in air bags, systems for extinguishing fire or as an additive in solid rocket propellants $[14,15]$. Carefully attention has also been given to salts of azotetrazole with metals $[5,11,12,16-18]$.

The synthesis of azotetrazoles occurred by the oxidation of 5-aminotetrazole with potassium permanganate $[19,20]$, by treating $N-R$-5-aminotetrazole $(\mathrm{R}=\mathrm{Me}, \mathrm{Ph}, \mathrm{tBu})$ with $\mathrm{N}$-bromosuccinimide (NBS) and azoisobutyronitrile (AIBN) in $\mathrm{CH}_{2} \mathrm{Cl}_{2}$ or $\mathrm{MeCN}$ [21] or by adding the amine to an aqueous acidic solution of sodium dichloroisocyanurate [22]. The diazenes with tetrazole and other substituent at nitrogen double bond were obtained by diazotization of aminotetrazole followed by coupling, with reasonable yields, between the diazoium salt and a suitable molecule at low temperature [23-25]. Although azulene has a weak nucleophilic character, it is enough reactive to interact with diazonium salts to produce diazenes. The reaction takes place regiospecifically at the azulene position 1(3), which has the highest electron density. Since the first azo-azulene obtained by Anderson Jr. [26], a large number of azulenes were obtained and a brief review of this class of compounds was recently published [27]. Our team has been concerned with the study of the synthesis and properties of diazenes

*email:lbirzan@yahoo.com 
with an azulene ring, unsubstituted or substituted with various aryl or heteroaryl groups, attached to azo bond (Scheme 1) [27]. In this context, it seemed interesting to study the diazenes with azulen-1-yl and tetrazol-5-yl systems attached at the azo bond. Supplementary, due to the involvement of the azulene moieties in several biologically active compounds [28], we considered that new such compounds with both azulene and tetrazole moieties in the same molecule could be of interest.

$$
\begin{aligned}
& \mathrm{Ar}=\text { phenyl (unsubstituted or substituted); naphthyl } \\
& \text { or } \\
& \text { pyridine and its } \mathrm{N} \text {-alkylated and } \mathrm{N} \text {-oxide derivatives } \\
& \text { 2-thiazole and its derivatives } \\
& \text { benzothiazoles and its derivatives } \\
& \text { 1,2,5-oxadiazol-3-yl and their } \mathrm{N} \text {-oxides }
\end{aligned}
$$

Scheme 1. Azulenes azocoupling

\section{Materials and methods}

\section{Materials}

Melting points (uncorrected) were measured with Kofler apparatus (Reichert Austria). Elemental analyses were performed using a Perkin Elmer CHN 240B analyzer. UV spectra were performed using a Varian Cary 100 spectrophotometer ( $\square$ values are given in $\mathrm{nm}$ and the molar extinction, $\varepsilon$, in $\mathrm{M}^{-1} \mathrm{~cm}^{-}$ ${ }^{1}$ units). ${ }^{1} \mathrm{H}$ and ${ }^{13} \mathrm{C}$ NMR: Bruker Avance DRX4 $\left({ }^{1} \mathrm{H}: 400 \mathrm{MHz},{ }^{13} \mathrm{C}: 100.62 \mathrm{MHz}\right)$ and Gemini 300 $\left({ }^{1} \mathrm{H}: 300 \mathrm{MHz},{ }^{13} \mathrm{C}: 75.47 \mathrm{MHz}\right.$ ), in $\mathrm{CDCl}_{3}$ or dimethylsulfoxide- $\mathrm{d}_{6}$ (DMSO-d $\mathrm{d}_{6}$ ); TMS was used as internal standard in $\mathrm{CDCl}_{3}$; several signals were assigned based on COSY, HETCOR and HMBC experiments. All compounds resulted as $(E)$ isomers. Mass spectra: Varian 1200L Triple Quadrupole LC/MS/MS spectrometer by direct injection in ESI. For column chromatography, silica gel 60 or alumina [II-III Brockmann grade, 70e230 mesh ASTM] were used. The dichloromethane (DCM) was distilled over $\mathrm{CaH}_{2}$ and the ether was stored over sodium. Acetonitrile $\left(\mathrm{CH}_{3} \mathrm{CN}\right)$ and tetrabutylammonium perchlorate (TBAP) from Fluka were used as received for solvent and supporting electrolyte. The electrochemical experiments were carried out using a potentiostat PGSTAT 12 AUTOLAB connected to a three-electrode cell. The working electrode was a glassy carbon electrode from Metrohm (diameter of $3 \mathrm{~mm}$ ). Its active surface was polished before each determination with diamond paste and was cleaned with the solvent. A platinum wire was used as auxiliary electrode, and the reference electrode was dependent on the solvent $(\mathrm{Ag} / \mathrm{Ag}+$ in acetonitrile or $\mathrm{Ag} / \mathrm{AgCl}$ in water). The electrochemical characterization of ligand was performed in $0.1 \mathrm{M} \mathrm{TBAP}, \mathrm{CH}_{3} \mathrm{CN} . \mathrm{Ag} / 10 \mathrm{mM}$ $\mathrm{AgNO}_{3}, 0.1 \mathrm{M}$ TBAP, $\mathrm{CH}_{3} \mathrm{CN}$ was used as reference electrode. The applied potential was finally referred to the potential of the ferrocene/ferrocenium redox couple $(\mathrm{Fc} / \mathrm{Fc}+)$ which in our experimental conditions was $-0.1 \mathrm{~V}$. Differential pulse voltammetry (DPV) curves were recorded at $0.01 \mathrm{~V} / \mathrm{s}$ with a pulse height of $0.025 \mathrm{~V}$ and a step time of $0.2 \mathrm{~s}$. The anodic and cathodic DPV scans have been recorded starting from the stationary potential.

\section{Diazotization and coupling}

5-Aminotetrazole $(210 \mathrm{mg}, 2 \mathrm{mmol})$ was dissolved in sulfuric acid $30 \%(5 \mathrm{~mL})$ and cooled at $0{ }^{\circ} \mathrm{C}$. At this solution a solution of $\mathrm{NaNO}_{2}(140 \mathrm{mg}, 2.03 \mathrm{mmol})$ in water $(1.4 \mathrm{~mL})$ was added and the resulted solution was kept with stirring at $0{ }^{\circ} \mathrm{C}$ for 15 minutes to generate the diazonium salt. This salt was added to a cooled solution of the azulene derivative $(2 \mathrm{mmols})$ in ethanol $(8 \mathrm{~mL})$ and pyridine $(3.4$ 
$\mathrm{mL}$ ) and stirred at $0{ }^{\circ} \mathrm{C}$. After stirring for 3 hours, the reaction mixture was diluted with water and acetic acid was added followed by the extraction with DCM. The organic layer was washed with water and was dried on $\mathrm{Na}_{2} \mathrm{SO}_{4}$. The solvent was evaporated at room temperature to near dryness. Petroleum ether was then added and the precipitated organic compound was filtered on a Buchner funnel. The ${ }^{1} \mathrm{H}-$ NMR spectrum allowed to estimate the yields reported in Scheme 2. For getting a pure analytical sample of compounds 1a-1e, small amount was chromatographed on silica gel using DCM-MeOH (alcohol $0 \%$ to $30 \%$ ). (Caution! Random, decomposition with nitrogen release took place when higher amount of material was placed on chromatography column). The solvent elimination was carried out at room temperature or bellow to prevent the products decomposition.

\section{Tetrazole benzylation}

Azulen-1-yldiazenyl tetrazole, 1a, $(122 \mathrm{mg}, 0.5 \mathrm{mmol})$ was treated with a solution of $\mathrm{NaOH}(20$ $\mathrm{mg}, 0.5 \mathrm{mmol})$ in ethanol $(1 \mathrm{~mL})$ and water $(0.5 \mathrm{~mL})$ and then benzyl bromide $(171 \mathrm{mg}, 1 \mathrm{mmol})$ was added. The reaction mixture was stirred over night at room temperature. Then DCM and water were added and a small amount of acetic acid needed to transfer the organic material to solvent. The crude mixture was analyzed on mass spectroscopy and was proved a mixture of mono and bis benzylated compounds. However, after chromatography on alumina only a brown-reddish material was obtained proved to be a mixture of 2- and 3-benzylated diazenes, $\mathbf{2}\left(\mathrm{PhCH}_{2}\right)$ and $\mathbf{3}\left(\mathrm{PhCH}_{2}\right)$. The repeated column chromatography on silica gel column using DCM-MeOH (increasing amounts of alcohol) as eluent allowed to obtain the diazene $\mathbf{2}\left(\mathrm{PhCH}_{2}\right)$ as pure product. With five times excess of benzyl bromide a small amount of monobenzylated compounds were obtained alongside with dibenzylated products $\mathbf{5}$ as bromide. The last products were obtained individually in very small amounts after repeated column chromatography and were characterized by mass and ${ }^{1} \mathrm{H}-\mathrm{NMR}$ spectra and elemental analysis. All solvent elimination was performed at room temperature.

\section{Tetrazole methylation}

Azulen-1-yldiazenyl tetrazole, 1a, $(244 \mathrm{mg}, 1 \mathrm{mmol})$ was reacted with dimethyl sulfate $(2 \mathrm{~mL})$ during 24 hours and then treated with a little ammonia solution. The mixture was poured in water (50 $\mathrm{mL})$ and methanol $(2 \mathrm{~mL})$ and some acetic acid was added. The organic layer was washed with water to remove the unreacted methyl sulfate, which decompose on column. The organic layer was dried on $\mathrm{Na}_{2} \mathrm{SO}_{4}$ and finally was concentrated to dryness in vacuum at room temperature. The reaction mixture was separate on a silica gel column (Caution! partial decomposition with nitrogen generation was random observed especially if methyl sulfate was still present). The first brown colored fraction, eluted with DCM was the mixture of mono methylated diazenes, $1 \mathrm{Me}$ and $2 \mathrm{Me}$, then, with a mixture of $\mathrm{DCM}-\mathrm{MeOH}(90 \%-10 \%)$, was eluted the starting material, 1a. Increasing the amount of methanol, the dimethylated diazene $\mathbf{8}$ as methyl sulfate salt was obtained.

\section{Interaction with metals}

a) Reaction with $\mathrm{Ag}^{+}$. To the solution of azulen-1-yldiazenyl tetrazole, $1 \mathbf{a}, 50 \mathrm{mg}(0.220 \mathrm{mmol})$ in $22 \mathrm{~mL}$ DCM was added solid $\mathrm{AgNO}_{3} 38 \mathrm{mg}(0.220 \mathrm{mmol})$ and $22 \mathrm{~mL} \mathrm{NaOH}$ solution $1 \mathrm{~N}(0.22$ $\mathrm{mmol}$ ) and the mixture was vigorously mixed $1 \mathrm{~h}$ at room temperature. The solvent was partially evaporated and $50 \mathrm{~mL}$ water was added to dissolve the unreacted silver nitrate. The precipitate formed was filtered and resulted $217 \mathrm{mg}$ solid, which was analyzed using mass spectrometry.

b) Metal dosing. Azulen-1-yldiazenyl tetrazole, 1a, $0.200 \mathrm{mg}(0.752 \mu \mathrm{mol})$ was dissolved in DCM $(25 \mathrm{~mL})$ leading to a brick-red solution $3 * 10^{-5} \mathrm{M}$. To this ligand solution, a DCM solution of $\mathrm{Fe}\left(\mathrm{ClO}_{4}\right)_{2} 3^{*} 10^{-3} \mathrm{M}$ is added gradually observing the color modification from brick-red to yelloworange and finally yellow or even yellow-green. First are added $2.5 \mathrm{~mL}(10 \mathrm{eq}$.) and then the amounts described in Figure 1 (15, 20, 25 eq., etc.). In the case of $\mathrm{Fe}\left(\mathrm{ClO}_{4}\right)_{3}$ a similar procedure was used by adding a $3^{*} 10^{-3} \mathrm{M}$ and the amounts are presented in the same table. The color variation is similar. 


\section{Products characterization}

(E)-5-(Azulen-1-yldiazenyl)-1H-tetrazole, 1a. Brown crystals, m.p. $242{ }^{\circ} \mathrm{C}$. UV-Vis, $\lambda_{\max }(\lg \varepsilon)$ : 226 (4.35), 273 (4.06), 295 (4.12), 336 (4.03), 433 (4.34) nm. ${ }^{1} \mathrm{H}-\mathrm{NMR}$ (DMSO-d 6 ), $\delta$ (ppm): 7.66 (d, $\left.{ }^{3} \mathrm{~J}=4.7 \mathrm{~Hz}, 1 \mathrm{H}, 3-\mathrm{H}\right), 7.76\left(\mathrm{t},{ }^{3} \mathrm{~J}=9.6 \mathrm{~Hz}, 1 \mathrm{H}, 5-\mathrm{H}\right), 7.87\left(\mathrm{t},{ }^{3} \mathrm{~J}=9.8 \mathrm{~Hz}, 1 \mathrm{H}, 7-\mathrm{H}\right), 8.14\left(\mathrm{t},{ }^{3} \mathrm{~J}=9.7\right.$ $\mathrm{Hz}, 1 \mathrm{H}, 6-\mathrm{H}), 8.37\left(\mathrm{~d},{ }^{3} \mathrm{~J}=4.7 \mathrm{~Hz}, 1 \mathrm{H}, 2-\mathrm{H}\right), 8.71\left(\mathrm{~d},{ }^{3} \mathrm{~J}=9.3 \mathrm{~Hz}, 1 \mathrm{H}, 4-\mathrm{H}\right), 9.44\left(\mathrm{t},{ }^{3} \mathrm{~J}=9.6 \mathrm{~Hz}, 1 \mathrm{H}\right.$, 8-H). ${ }^{13}$ C-NMR (DMSO-d 6 ), $\delta$ (ppm): 123.2, 124.5, 127.3, 131.0, 131.1, 136.3, 140.8, 142.1, 143.8, 146.4, 164.7. IR (neat): 742.5, 755.8, 771.8, 788.0, 878.9, 1016.4, 1037.6, 1053.8, 1100.4, 1115.1, 1125.4, 1125.4, 1164.1, 1217.9, 1254.5 M, 1314.3, 1328.4, 1360.3, 1404.9, 1416.2, 1442.1, 1454.8, 1487.9, 1533.7, 1573.5 cm-1. MS [ESI]: 225 [M+1]. Calc. for $\mathrm{C}_{11} \mathrm{H}_{8} \mathrm{~N}_{6}: \mathrm{C}, 58.92 ; \mathrm{H}, 3.60 ; \mathrm{N}, 37.48$. Found: C, 58.91; H, 3.62; N, 37.47.

(E)-5-((4,6,8-Trimethylazulen-1-yl)diazenyl)-1H-tetrazole, 1b. Brown crystals, m.p. $275{ }^{\circ} \mathrm{C}(\mathrm{dec})$. UV-Vis, $\lambda_{\max }(\lg \varepsilon): 234$ (4.36), 256sh (4.23), 315 (4.14), 332 (4.07), 334 (4.07), 336 (4.07), 435 (4.34) nm. ${ }^{1} \mathrm{H}-\mathrm{NMR}\left(\mathrm{DMSO}_{\mathrm{d}}\right), \delta(\mathrm{ppm}): 2.66(\mathrm{~s}, 3 \mathrm{H}, \mathrm{Me}), 2.85$ (s, $\left.3 \mathrm{H}, \mathrm{Me}\right), 3.23(\mathrm{~s}, 3 \mathrm{H}, \mathrm{Me}), 7.46\left(\mathrm{~d},{ }^{3} \mathrm{~J}\right.$ $=4.9 \mathrm{~Hz}, 1 \mathrm{H}, 3-\mathrm{H}), 7.60(\mathrm{~s}, 1 \mathrm{H}, 5-\mathrm{H}), 7.67(\mathrm{~s}, 1 \mathrm{H}, 7-\mathrm{H}), 7.99\left(\mathrm{~d},{ }^{3} \mathrm{~J}=4.9 \mathrm{~Hz}, 1 \mathrm{H}, 2-\mathrm{H}\right) .{ }^{13} \mathrm{C}-\mathrm{NMR}$ (DMSO-d $), \delta$ (ppm): 25.05, 27.93, 29.12, 120.7, 121.5, 134.6, 136.6, 136.7, 143.4, 148.0, 149.5, 150.7, 151.1, 165.6. IR (neat): 700.3, 725.7, 749.6, 803.8, 860.5, 875.8, 930.1, 1036.1, 1051.7, 1077.3, 1109.8, 1191.1, 1208.9, 1234.4, 1285.6 M, 1354.6, 1370.3, 1388.2, 1440.6, 1498.4, 1547.8, 1581.4, 1622.0, 2965.4, 2983.9, $3098.7 \mathrm{~cm}^{-1}$. MS [ESI]: 267 [M+1]. Calc. for $\mathrm{C}_{14} \mathrm{H}_{11} \mathrm{~N}_{6}: \mathrm{C}, 63.14 ; \mathrm{H}, 5.30 ; \mathrm{N}$, 31.56. Found: C, 63.15; H, 5.31; N, 31.55.

(E)-5-((5-Isopropyl-3,8-dimethylazulen-1-yl)diazenyl)-1H-tetrazole 1c. Black crystals, m. p. 303 ${ }^{\circ} \mathrm{C}$. UV-Vis, $\lambda_{\max }(\lg \varepsilon): 231$ (4.37), $253 \mathrm{sh}(4.28), 311$ (4.08), 313 (4.08), 347 (4.05), 350 (4.02), 471 (4.35) nm. ${ }^{1} \mathrm{H}-\mathrm{NMR}$ (DMSO-d ${ }_{6}$ ), $\delta(\mathrm{ppm}): 1.29$ (d, $\left.{ }^{3} \mathrm{~J}=6.9 \mathrm{~Hz}, 6-\mathrm{H}, \mathrm{CHMe}\right), 2.47$ (s, $\left.3 \mathrm{H}, \mathrm{Me}\right), 3.13$ (sept, $1 \mathrm{H}, \mathrm{CHMe}), 3.14$ (s, $3 \mathrm{H}, \mathrm{Me}), 7.61\left(\mathrm{~d},{ }^{3} \mathrm{~J}=10.7 \mathrm{~Hz}, 1 \mathrm{H}, 7-\mathrm{H}\right), 7.74\left(\mathrm{~d},{ }^{3} \mathrm{~J}=11.0 \mathrm{~Hz}, 1 \mathrm{H}, 6-\right.$ H). ${ }^{13} \mathrm{C}-\mathrm{NMR}\left(\mathrm{DMSO}_{\mathrm{d}}\right.$ ), $\delta(\mathrm{ppm}): 12.79,23.95,28.11,37.36,124.6,131.3,136.0,136.3,137.9$, 138.7, 145.2, 145.8, 150.2, 150.4, 164.4. IR (neat): 437.2, 629.2, 823.5, 851.4, 870.2, 937.5, 967.3, 1037.0, 1101.1, 1164.7, 1232.4, 1262.1 M, 1340.9, 1389.2, 1403.8, 1433.2, 1500.4, 1552.7, 1585.3, 1618.1, 2073.2, 2337.9, 2964.2 $\mathrm{cm}^{-1}$. MS [ESI]: 295 [M+1]. Calc. for $\mathrm{C}_{16} \mathrm{H}_{18} \mathrm{~N}_{6}: \mathrm{C}, 65.29 ; \mathrm{H}, 6.16 ; \mathrm{N}$, 28.55. Found: C, 65.27; H, 6.17; N, 28.56.

(E)-5-((6-Tert-butyl-4,8-dimethylazulen-1-yl)diazenyl)-1H-tetrazole, 1d. Brown crystals, m. p. 287 ${ }^{\circ} \mathrm{C}$ (dec). UV-Vis, $\lambda_{\max }(\lg \varepsilon): 234$ (4.32), 262sh (4.18), 314 (4.13), 334 (4.04), 341 (4.03), 435 (4.30) nm. ${ }^{1} \mathrm{H}-\mathrm{NMR}$ (DMSO-d D $, \delta(\mathrm{ppm}): 1.47\left(\mathrm{~s}, 9 \mathrm{H}, \mathrm{CMe}_{3}\right), 2.95$ (s, $\left.3 \mathrm{H}, \mathrm{Me}\right), 3.33$ (s, $\left.3 \mathrm{H}, \mathrm{Me}\right), 7.50(\mathrm{~d}$, $\left.{ }^{3} \mathrm{~J}=5.0 \mathrm{~Hz}, 1 \mathrm{H}, 3-\mathrm{H}\right), 7.83(\mathrm{~s}, 1 \mathrm{H}, 5-\mathrm{H}), 7.92(\mathrm{~s}, 1 \mathrm{H}, 7-\mathrm{H}), 8.05\left(\mathrm{~d},{ }^{3} \mathrm{~J}=5.0 \mathrm{~Hz}, 1 \mathrm{H}, 2-\mathrm{H}\right) .{ }^{13} \mathrm{C}-\mathrm{NMR}$ $\left(\right.$ DMSO-d $\left._{6}\right), \delta$ (ppm): 25.65, 29.66, 31.42, 39.03, 120.6, 122.2, 131.5, 133.6, 136.9, 143.8, 147.8, 149.5, 151.1, 162.1, 165.5. IR (neat): 728.1, 799.2, 876.2, 919.9, 1045.7, 1072.7, 1117.8, 1206.0, 1234.8, 1275.4 M, 1318.3, 1365.9, 1386.6, 1438.9, 1495.3, 1527.9, 1576.2, 2964.2, $3115.9 \mathrm{~cm}^{-1} . \mathrm{MS}$ [ESI]: 309 [M+1]. Calc. for $\mathrm{C}_{17} \mathrm{H}_{20} \mathrm{~N}_{6}$ : C, 66.21; H, 6.54; N, 27.25. Found: C, 66.22; H, 6.52; N, 27.26.

(E)-5-((6,8-Dimethyl-3,4-dihydrocyclopenta[cd]azulen-1-yl)diazenyl)-1H-tetrazole,1e. Brown crystals, m. p. $292{ }^{\circ} \mathrm{C}$. UV-Vis, $\lambda_{\max }(\lg \varepsilon): 233,250 \mathrm{sh}, 296,321,348,473 \mathrm{~nm} .{ }^{1} \mathrm{H}-\mathrm{NMR}$ (DMSO-d 6 ), $\delta$ (ppm): 2.57 (s, $3 \mathrm{H}, \mathrm{Me}$ ), 3.01 (s, $3 \mathrm{H}, \mathrm{Me}$ ), 3.10-3.12 (m, $2 \mathrm{H}, \mathrm{Et}), 3.56-3.59$ (m, $2 \mathrm{H}, \mathrm{Et}), 7.36$ (bs, 1 $\mathrm{H}, 5-\mathrm{H}), 7.37$ (d, $\left.{ }^{3} \mathrm{~J}=4.9 \mathrm{~Hz}, 1 \mathrm{H}, 2-\mathrm{H}\right), 7.43$ (bs, $\left.1 \mathrm{H}, 7-\mathrm{H}\right) .{ }^{13} \mathrm{C}-\mathrm{NMR}$ (DMSO-d 6 ), $\delta$ (ppm): 24.78, 26.20, 28.49, 38.87, 112.0, 125.7, 128.5, 131.7, 135.7, 145.2, 150.3, 151.3, 152.3, 152.4, 159.1, 164.2. IR (neat): 539, 589, 650, 683, 712, 845, 982, 1028, 1100, 1131, 1184, 1229, 1264, 1351, 1412, 1444, 1471, 1499, 1572, 1590, 2018, 2095, 2173, 2200, 2333, 2363, 2728, 2913, 3065, $3383 \mathrm{~cm}^{-1}$. MS [ESI]: 279 [M+1]. Calc. for $\mathrm{C}_{15} \mathrm{H}_{14} \mathrm{~N}_{6}$ : C, 64.73; H, 5.07; N, 30.20. Found: C, 64.70; H, 5.09; N, 30.21.

(E)-5-(Azulen-1-yldiazenyl)-2-benzyl-2H-tetrazole, 2( $\left.\mathrm{PhCH}_{2}\right)$. Brown crystals, m. p. $134{ }^{\circ} \mathrm{C}$. UVVis, $\lambda_{\max }(\lg \varepsilon): 224$ (4.39), 284 (4.02), 291 (4.11), 337 (4.11), 433 (4.54) nm. ${ }^{1}$ H-NMR (acetone-d 6 ), $\delta$ (ppm): 6.01 (s, $\left.2 \mathrm{H}, \mathrm{PhCH}_{2}\right), 7.42$ (t, $\left.{ }^{3} \mathrm{~J}=7.9 \mathrm{~Hz}, 2 \mathrm{H}, 3^{\prime}-\mathrm{H}, 5^{\prime}-\mathrm{H}\right), 7.44$ (t, $\left.{ }^{3} \mathrm{~J}=7.9 \mathrm{~Hz}, 1 \mathrm{H}, 4^{\prime}-\mathrm{H}\right)$, $7.51\left(\mathrm{bd},{ }^{3} \mathrm{~J}=7.9 \mathrm{~Hz}, 2 \mathrm{H}, 2^{\prime}-\mathrm{H}, 6{ }^{\prime}-\mathrm{H}\right), 7.59\left(\mathrm{~d},{ }^{3} \mathrm{~J}=4.7 \mathrm{~Hz}, 1 \mathrm{H}, 3-\mathrm{H}\right), 7.67\left(\mathrm{t},{ }^{3} \mathrm{~J}=9.6 \mathrm{~Hz}, 1 \mathrm{H}, 5-\mathrm{H}\right)$, $7.78\left(\mathrm{t},{ }^{3} \mathrm{~J}=9.8 \mathrm{~Hz}, 1 \mathrm{H}, 7-\mathrm{H}\right), 8.05\left(\mathrm{t},{ }^{3} \mathrm{~J}=9.7 \mathrm{~Hz}, 1 \mathrm{H}, 6-\mathrm{H}\right), 8.26\left(\mathrm{~d},{ }^{3} \mathrm{~J}=4.6 \mathrm{~Hz}, 1 \mathrm{H}, 2-\mathrm{H}\right), 8.64(\mathrm{~d}$, $\left.{ }^{3} \mathrm{~J}=9.3 \mathrm{~Hz}, 1 \mathrm{H}, 4-\mathrm{H}\right), 9.35\left(\mathrm{t},{ }^{3} \mathrm{~J}=9.8 \mathrm{~Hz}, 1 \mathrm{H}, 8-\mathrm{H}\right) .{ }^{13} \mathrm{C}-\mathrm{NMR}$ (acetone-d $\left.{ }_{6}\right), \delta(\mathrm{ppm}): 57.43,122.1$, 
125.3, 129.0, 129.2, 129.3, 129.5, 129.8, 136.3, 137.4, 140.4, 141.6, 148.7, 149.4, 172.4. IR (neat): 562, 580, 686, 701, 727, 746, 782, 1010, 1043, 1216, 1261, 1310, 1325, 1357, 1413, 1432, 1450, 1439, 1493, 1572, 1724, 2027, 2048, 2180, 2333, 2362, $3032 \mathrm{~cm}^{-1}$. MS [ESI]: 315 [M+1]. Calc. for $\mathrm{C}_{18} \mathrm{H}_{14} \mathrm{~N}_{6}$ : C, 64.73; H, 5.07; N, 30.20. Found: C, 64.72; H, 5.07; N, 30.21.

(E)-5-(azulen-1-yldiazenyl)-1-benzyl-1H-tetrazole, 1( $\left.\mathrm{PhCH}_{2}\right) .{ }^{1} \mathrm{H}-\mathrm{NMR}$ (acetone- $\left.\mathrm{d}_{6}\right), \delta(\mathrm{ppm})$ : 5.99 (s, 2 H, PhCH $H_{2}$, 7.35-7.50 (m, 4 H, 3'-H, 4'-H, 5'-H), 7.55 (bd, 3' $=7.9$ Hz, 4 H, 2'-H, 6'-H), $7.66\left(\mathrm{~d},{ }^{3} \mathrm{~J}=4.7 \mathrm{~Hz}, 1 \mathrm{H}, 3-\mathrm{H}\right), 7.78\left(\mathrm{t},{ }^{3} \mathrm{~J}=9.6 \mathrm{~Hz}, 1 \mathrm{H}, 5-\mathrm{H}\right), 7.85\left(\mathrm{t},{ }^{3} \mathrm{~J}=9.8 \mathrm{~Hz}, 1 \mathrm{H}, 7-\mathrm{H}\right), 8.13\left(\mathrm{t},{ }^{3} \mathrm{~J}\right.$ $=9.7 \mathrm{~Hz}, 1 \mathrm{H}, 6-\mathrm{H}), 8.39\left(\mathrm{~d},{ }^{3} \mathrm{~J}=4.6 \mathrm{~Hz}, 1 \mathrm{H}, 2-\mathrm{H}\right), 8.68\left(\mathrm{~d},{ }^{3} \mathrm{~J}=9.3 \mathrm{~Hz}, 1 \mathrm{H}, 4-\mathrm{H}\right), 9.31\left(\mathrm{t},{ }^{3} \mathrm{~J}=9.8\right.$ $\mathrm{Hz}, 1 \mathrm{H}, 8-\mathrm{H})$. In mixture with $2\left(\mathrm{PhCH}_{2}\right)$.

(E)-5-(Azulen-1-yldiazenyl)-2,3-dibenzyl-2H-tetrazol-3-ium bromide, 5(2,3). ${ }^{1} \mathrm{H}-\mathrm{NMR}$ (acetone$\left.\mathrm{d}_{6}\right), \delta(\mathrm{ppm}): 6.21\left(\mathrm{~s}, 4 \mathrm{H}, 2 \mathrm{PhCH}_{2}\right), 7.45\left(\mathrm{t},{ }^{3} \mathrm{~J}=7.9 \mathrm{~Hz}, 4 \mathrm{H}, 3^{\prime}-\mathrm{H}, 5^{\prime}-\mathrm{H}\right), 7.42\left(\mathrm{t},{ }^{3} \mathrm{~J}=7.9 \mathrm{~Hz}, 2 \mathrm{H}, 4^{\prime}-\right.$ H), $7.63\left(\mathrm{bd},{ }^{3} \mathrm{~J}=7.9 \mathrm{~Hz}, 4 \mathrm{H}, 2^{\prime}-\mathrm{H}, 66^{\prime}-\mathrm{H}\right), 7.95\left(\mathrm{~d},{ }^{3} \mathrm{~J}=4.7 \mathrm{~Hz}, 1 \mathrm{H}, 3-\mathrm{H}\right), 8.26\left(\mathrm{t},{ }^{3} \mathrm{~J}=9.6 \mathrm{~Hz}, 1 \mathrm{H}, 5-\right.$ $\mathrm{H}), 8.33\left(\mathrm{t},{ }^{3} \mathrm{~J}=9.8 \mathrm{~Hz}, 1 \mathrm{H}, 7-\mathrm{H}\right), 8.51\left(\mathrm{t},{ }^{3} \mathrm{~J}=9.7 \mathrm{~Hz}, 1 \mathrm{H}, 6-\mathrm{H}\right), 8.47\left(\mathrm{~d},{ }^{3} \mathrm{~J}=4.7 \mathrm{~Hz}, 1 \mathrm{H}, 2-\mathrm{H}\right), 8.99$ $\left(\mathrm{d},{ }^{3} \mathrm{~J}=9.3 \mathrm{~Hz}, 1 \mathrm{H}, 4-\mathrm{H}\right), 9.39\left(\mathrm{t},{ }^{3} \mathrm{~J}=9.8 \mathrm{~Hz}, 1 \mathrm{H}, 8-\mathrm{H}\right) . \mathrm{MS}$ [ESI]: $406\left[\mathrm{M}^{+}+1\right]$.

(E)-5-(Azulen-1-yldiazenyl)-1,3-dibenzyl-1H-tetrazol-3-ium bromide, 5(1,3). ${ }^{1} \mathrm{H}-\mathrm{NMR}$ (acetone$\left.\mathrm{d}_{6}\right), \delta(\mathrm{ppm}): 5.99\left(\mathrm{~s}, 2 \mathrm{H}, \mathrm{PhCH}_{2} \mathrm{~N}\right), 6.16$ (s, $\left.2 \mathrm{H}, \mathrm{PhCH}_{2} \mathrm{~N}^{+}\right), 7.30-7.38$ (m, $6 \mathrm{H}, 3$ '-H, 3'-H, 5'-H, 5'$\mathrm{H}), 7.42\left(\mathrm{t},{ }^{3} \mathrm{~J}=7.9 \mathrm{~Hz}, 2 \mathrm{H}, 4^{\prime}-\mathrm{H}\right), 7.43\left(\mathrm{bd},{ }^{3} \mathrm{~J}=7.9 \mathrm{~Hz}, 4 \mathrm{H}, 2^{\prime}-\mathrm{H}, 6{ }^{\prime}-\mathrm{H}\right), 7.60\left(\mathrm{~d},{ }^{3} \mathrm{~J}=4.7 \mathrm{~Hz}, 1 \mathrm{H}, 3-\right.$ $\mathrm{H}), 7.66\left(\mathrm{t},{ }^{3} \mathrm{~J}=9.6 \mathrm{~Hz}, 1 \mathrm{H}, 5-\mathrm{H}\right), 7.84\left(\mathrm{t},{ }^{3} \mathrm{~J}=9.8 \mathrm{~Hz}, 1 \mathrm{H}, 7-\mathrm{H}\right), 8.05\left(\mathrm{t},{ }^{3} \mathrm{~J}=9.7 \mathrm{~Hz}, 1 \mathrm{H}, 6-\mathrm{H}\right), 8.33$ $\left(\mathrm{d},{ }^{3} \mathrm{~J}=4.7 \mathrm{~Hz}, 1 \mathrm{H}, 2-\mathrm{H}\right), 8.64\left(\mathrm{~d},{ }^{3} \mathrm{~J}=9.3 \mathrm{~Hz}, 1 \mathrm{H}, 4-\mathrm{H}\right), 9.20\left(\mathrm{t},{ }^{3} \mathrm{~J}=9.8 \mathrm{~Hz}, 1 \mathrm{H}, 8-\mathrm{H}\right) . \mathrm{MS}[\mathrm{ESI}]$ : $406\left[\mathrm{M}^{+}+1\right]$.

(E)-5-(Azulen-1-yldiazenyl)-1,4-dibenzyl-1H-tetrazol-4-ium bromide, 5(1,4). ${ }^{1} \mathrm{H}-\mathrm{NMR}$ (DMSO$\mathrm{d}_{6}$ ), $\delta$ (ppm): 6.03 (s, $\left.4 \mathrm{H}, 2 \mathrm{PhCH}_{2}\right), 7.42$ (t, $\left.{ }^{3} \mathrm{~J}=7.3 \mathrm{~Hz}, 2 \mathrm{H}, 4^{\prime}-\mathrm{H}\right), 7.43$ (t, ${ }^{3} \mathrm{~J}=7.3 \mathrm{~Hz}, 4 \mathrm{H}, 3^{\prime}-\mathrm{H}, 5^{\prime}-$ $\mathrm{H}), 7.53\left(\mathrm{~d},{ }^{3} \mathrm{~J}=7.3 \mathrm{~Hz}, 4 \mathrm{H}, 2^{\prime}-\mathrm{H}, 6{ }^{\prime}-\mathrm{H}\right), 7.94\left(\mathrm{~d},{ }^{3} \mathrm{~J}=5.0 \mathrm{~Hz}, 1 \mathrm{H}, 3-\mathrm{H}\right), 8.33\left(\mathrm{~d},{ }^{3} \mathrm{~J}=4.9 \mathrm{~Hz}, 1 \mathrm{H}, 2-\right.$ $\mathrm{H}), 8.33\left(\mathrm{t},{ }^{3} \mathrm{~J}=9.6 \mathrm{~Hz}, 1 \mathrm{H}, 5-\mathrm{H}\right), 8.39\left(\mathrm{t},{ }^{3} \mathrm{~J}=9.8 \mathrm{~Hz}, 1 \mathrm{H}, 7-\mathrm{H}\right), 8.54\left(\mathrm{t},{ }^{3} \mathrm{~J}=9.7 \mathrm{~Hz}, 1 \mathrm{H}, 6-\mathrm{H}\right), 8.88$ $\left(\mathrm{d},{ }^{3} \mathrm{~J}=9.3 \mathrm{~Hz}, 1 \mathrm{H}, 4-\mathrm{H}\right), 8.98\left(\mathrm{t},{ }^{3} \mathrm{~J}=9.8 \mathrm{~Hz}, 1 \mathrm{H}, 8-\mathrm{H}\right)$. MS [ESI]: $406\left[\mathrm{M}^{+}+1\right]$.

(E)-5-(Azulen-1-yldiazenyl)-1-methyl-1H-tetrazole, 1Me. Brown crystals, m. p. $198 \mathrm{C}$. UVVis, $\lambda_{\max }$ (lg $\varepsilon$ ): 222 (4.35), 295 (4.12), 339 (4.03), 448 (4.36) nm. ${ }^{1} \mathrm{H}-\mathrm{NMR}\left(\mathrm{CDCl}_{3}\right), \delta$ (ppm): 4.33 (s, $3 \mathrm{H}, \mathrm{Me}), 7.50\left(\mathrm{~d},{ }^{3} \mathrm{~J}=5.1 \mathrm{~Hz}, 1 \mathrm{H}, 3-\mathrm{H}\right), 7.61\left(\mathrm{t},{ }^{3} \mathrm{~J}=9.6 \mathrm{~Hz}, 1 \mathrm{H}, 5-\mathrm{H}\right), 7.72\left(\mathrm{t},{ }^{3} \mathrm{~J}=9.8 \mathrm{~Hz}, 1 \mathrm{H}, 7-\right.$ $\mathrm{H}), 7.95\left(\mathrm{t},{ }^{3} \mathrm{~J}=9.7 \mathrm{~Hz}, 1 \mathrm{H}, 6-\mathrm{H}\right), 8.32\left(\mathrm{~d},{ }^{3} \mathrm{~J}=4.6 \mathrm{~Hz}, 1 \mathrm{H}, 2-\mathrm{H}\right), 8.46\left(\mathrm{~d},{ }^{3} \mathrm{~J}=9.3 \mathrm{~Hz}, 1 \mathrm{H}, 4-\mathrm{H}\right), 9.45$ $\left(\mathrm{t},{ }^{3} \mathrm{~J}=9.8 \mathrm{~Hz}, 1 \mathrm{H}, 8-\mathrm{H}\right) .{ }^{13} \mathrm{C}-\mathrm{NMR}\left(\mathrm{CDCl}_{3}\right), \delta(\mathrm{ppm}): 34.40,122.9,125.6,127.3,130.4,130.8,136.9$, 139.7, 141.1, 144.4, 148.8, 164.7. MS [ESI]: 239 [M+1]. Calc. for $\mathrm{C}_{12} \mathrm{H}_{10} \mathrm{~N}_{6}: \mathrm{C}, 60.50 ; \mathrm{H}, 4.23 ; \mathrm{N}$, 35.27. Found: C, 60.52; H, 4.25; N, 35.23.

(E)-5-(Azulen-1-yldiazenyl)-2-methyl-1H-tetrazole, 2Me. ${ }^{1} \mathrm{H}-\mathrm{NMR}\left(\mathrm{CDCl}_{3}\right), \delta(\mathrm{ppm}): 4.46(\mathrm{~s}, 3 \mathrm{H}$, $\mathrm{Me}), 7.47\left(\mathrm{~d},{ }^{3} \mathrm{~J}=5.1 \mathrm{~Hz}, 1 \mathrm{H}, 3-\mathrm{H}\right), 7.52\left(\mathrm{t},{ }^{3} \mathrm{~J}=9.6 \mathrm{~Hz}, 1 \mathrm{H}, 5-\mathrm{H}\right), 7.57\left(\mathrm{t},{ }^{3} \mathrm{~J}=9.8 \mathrm{~Hz}, 1 \mathrm{H}, 7-\mathrm{H}\right)$, $7.87\left(\mathrm{t},{ }^{3} \mathrm{~J}=9.7 \mathrm{~Hz}, 1 \mathrm{H}, 6-\mathrm{H}\right), 8.41\left(\mathrm{~d},{ }^{3} \mathrm{~J}=4.6 \mathrm{~Hz}, 1 \mathrm{H}, 2-\mathrm{H}\right), 8.42\left(\mathrm{~d},{ }^{3} \mathrm{~J}=9.3 \mathrm{~Hz}, 1 \mathrm{H}, 4-\mathrm{H}\right), 9.45(\mathrm{t}$, $\left.{ }^{3} \mathrm{~J}=9.8 \mathrm{~Hz}, 1 \mathrm{H}, 8-\mathrm{H}\right)$. In mixture with $3 \mathrm{Me}$.

(E)-5-(Azulen-1-yldiazenyl)-1,4-dimethyl-1H-tetrazol-1-ium methylsulfate, 6. Brown crystals m. p. $190 \mathrm{C}$ (dec). UV-Vis, $\lambda_{\max }(\lg \varepsilon): 219$ (4.64), 247 (4.15), 308 (3.94), 328 (3.91), 517 (4.39) nm. ${ }^{1} \mathrm{H}-$ NMR (DMSO-d 6 ), $\delta(\mathrm{ppm}): 4.36(\mathrm{~s}, 6 \mathrm{H}, \mathrm{Me}), 7.94\left(\mathrm{~d},{ }^{3} \mathrm{~J}=5.1 \mathrm{~Hz}, 1 \mathrm{H}, 3-\mathrm{H}\right), 8.33\left(\mathrm{~d},{ }^{3} \mathrm{~J}=4.6 \mathrm{~Hz}, 1\right.$ $\mathrm{H}, 2-\mathrm{H}), 8.36\left(\mathrm{t},{ }^{3} \mathrm{~J}=9.6 \mathrm{~Hz}, 1 \mathrm{H}, 5-\mathrm{H}\right), 8.43\left(\mathrm{t},{ }^{3} \mathrm{~J}=9.8 \mathrm{~Hz}, 1 \mathrm{H}, 7-\mathrm{H}\right), 8.57\left(\mathrm{t},{ }^{3} \mathrm{~J}=9.7 \mathrm{~Hz}, 1 \mathrm{H}, 6-\mathrm{H}\right)$, $9.00\left(\mathrm{~d},{ }^{3} \mathrm{~J}=9.3 \mathrm{~Hz}, 1 \mathrm{H}, 4-\mathrm{H}\right), 9.41\left(\mathrm{t},{ }^{3} \mathrm{~J}=9.8 \mathrm{~Hz}, 1 \mathrm{H}, 8-\mathrm{H}\right)$. MS [ESI]: $253\left[\mathrm{M}^{+}+1\right]$. Calc. for $\mathrm{C}_{14} \mathrm{H}_{16} \mathrm{~N}_{6} \mathrm{O}_{4} \mathrm{~S}$ : C, 46.15; H, 4.43; N, 23.06. Found: C, 46.12; H, 4.45; N, 23.03.

\section{Results and discussions Syntheses}

Diazotization and coupling. The diazotization of 5-aminotetrazole takes place under $-10{ }^{\circ} \mathrm{C}$ and large excess of sulfuric or phosphoric acid [21-23] was used in the aim to prevent the possible explosion (Caution! in concentrate solutions it can decompose violently). For the subsequent coupling, azulenes were dissolved in a mixture of pyridine and alcohol and treated at $0{ }^{\circ} \mathrm{C}$ with the above obtained solution of the diazonium salt. 5-substituted tetrazoles unsubstituted at nitrogen atoms, can 
exist in two tautomeric forms, which are in equilibrium in solutions as shown in Scheme 3. As will be outlined below, the generated diazenes have a tendency to ionize both in basic and in acidic medium giving salts, which are soluble in the aqueous medium. Therefore, for access to the reaction product, the reaction mixture was diluted with water, then acetic acid was added and the product was extracted with methylene chloride (DCM). Acidulation afforded a buffered acetic acid/acetate medium; the compounds reach their isoelectric point and become less soluble in water and more soluble in organic solvents. For a further purification, small samples were chromatographed on silica gel using DCM$\mathrm{MeOH}$ with increasing amount of alcohol (0-30\%). Unfortunately, the chromatography occurs with partial products degradation, especially if the column is longer. (Random, decomposition with nitrogen release took place when a higher amount of material was placed on chromatography column). The solvent elimination was carried out at room temperature or bellow to prevent the products decomposition. The generality of the sequence diazotization-coupling and the good resulted yields were highlighted using different azulenes (Scheme 2).

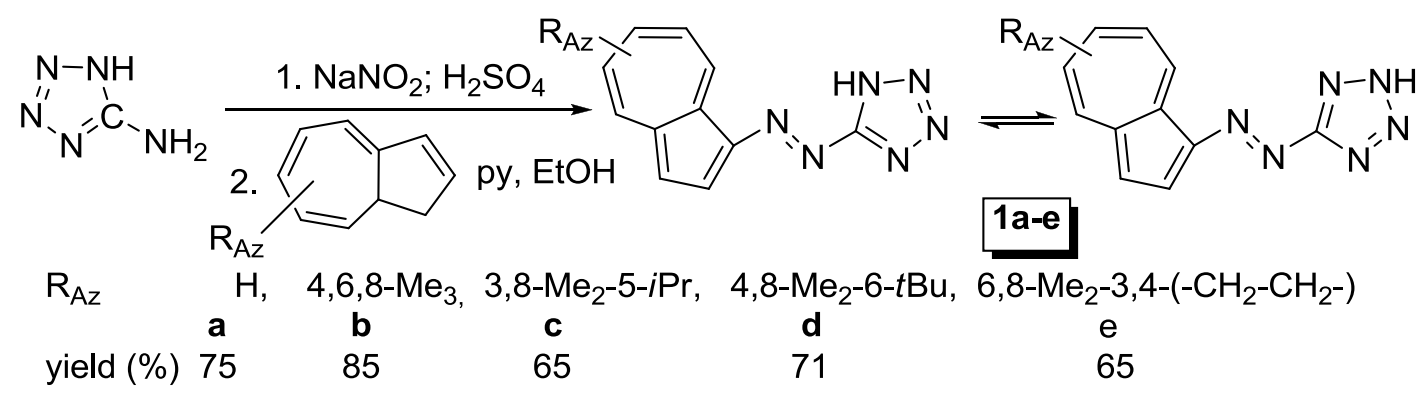

Scheme 2. Diazotization of 5-aminotetrazole and azocoupling of diazonium salt with azulenes

Tetrazole alkylation. The 5-aminotetrazoles supplementary substituted with alkyl or aryl by treatment with sodium nitrite generates nitrosamines and therefore the azo coupling reaction fails [29]. Therefore, tetrazole diazenes with alkyl at heterocyclic nitrogen can be obtained only by alkylation. One of the most important properties of the tetrazole system results from its aromatic $\pi$-electronic system and the lone pair on each nitrogen. Thus, this heteroaromatic ring can be protonated, functionalized or coordinated. One of the most studied reactions was the alkylation of this system and several data were provided about the alkylation of azotetrazole [4,8,9,12]. The starting materials for this reaction can be the neutral azotetrazole, its salts or even complexes with different metals. It is known that the electrophile alkylations of 5-substituted tetrazoles are not selective and occur at the position 1 and/or $2[8,9]$.

There are many examples leading to the idea that increasing the electron-withdrawing properties of substituent at position 5 favors the formation of isomer 2-substituted rather than that 1-substituted [6]. In this regard, the results reported by Efimova on the methylation of 5-phenyltetrazoles with dimethyl sulfate are interesting [30,31]. Whereas in the presence of trimethylamine the ratio between the obtained 1- and 2- methyl isomers in different solvents varied in the range 0.31-0.38, in excess of dimethyl sulfate without basic amines reaction led to an equimolecular mixture of isomers. The equimolar isomers ratio gave also the benzylation of 5-benzyltetrazole [32]. At the alkylation of 5(azulen-1-yldiazenyl)tetrazoles also resulted 1- and 2- substituted isomers with a ratio between them influenced by the alkylating agent and reaction conditions (Scheme 3). The electron donor ability of azulen-1-yl moiety seems to have little influence on the products ratio but more significant on the yields. 


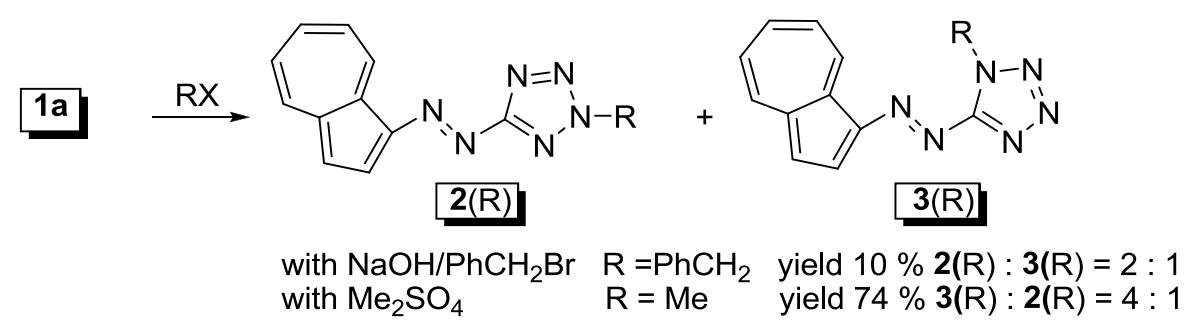

Scheme 3. Alkylation of 5-(azulen-1-yldiazenyl)tetrazole

The benzylation of diazene was realized using the common alkylation in alkaline medium. The intermediate tetrazolate anions 4 (Scheme 4) was treated in situ with excess of benzyl bromide. The electron donor effect of azulen-1-yl moiety decreases in some extent the acidity of compounds $\mathbf{1}$ and hinders the generation of intermediate 4 thus lowering the yield of benzylation. By the acidulation (acetic acid), a buffered medium was also achieved in the aim to decrease the solubility in water of products. Because a good separation between the benzylated products $2\left(\mathrm{PhCH}_{2}\right)$ and $\mathbf{3}\left(\mathrm{PhCH}_{2}\right)$ failed and only the major compound $\mathbf{2}\left(\mathrm{PhCH}_{2}\right)$ could be individually separated, the ratio between the isomers was estimated from the proton spectrum of products mixture before their separation. Together with mono benzylated products, the alkylation produces also bis alkylated compounds at the heterocycle, obviously as salts 5. Despite the increases in some extent of salts amount when a large excess of alkylating reagent was used, the very low amounts prevented the complete characterization of salts.
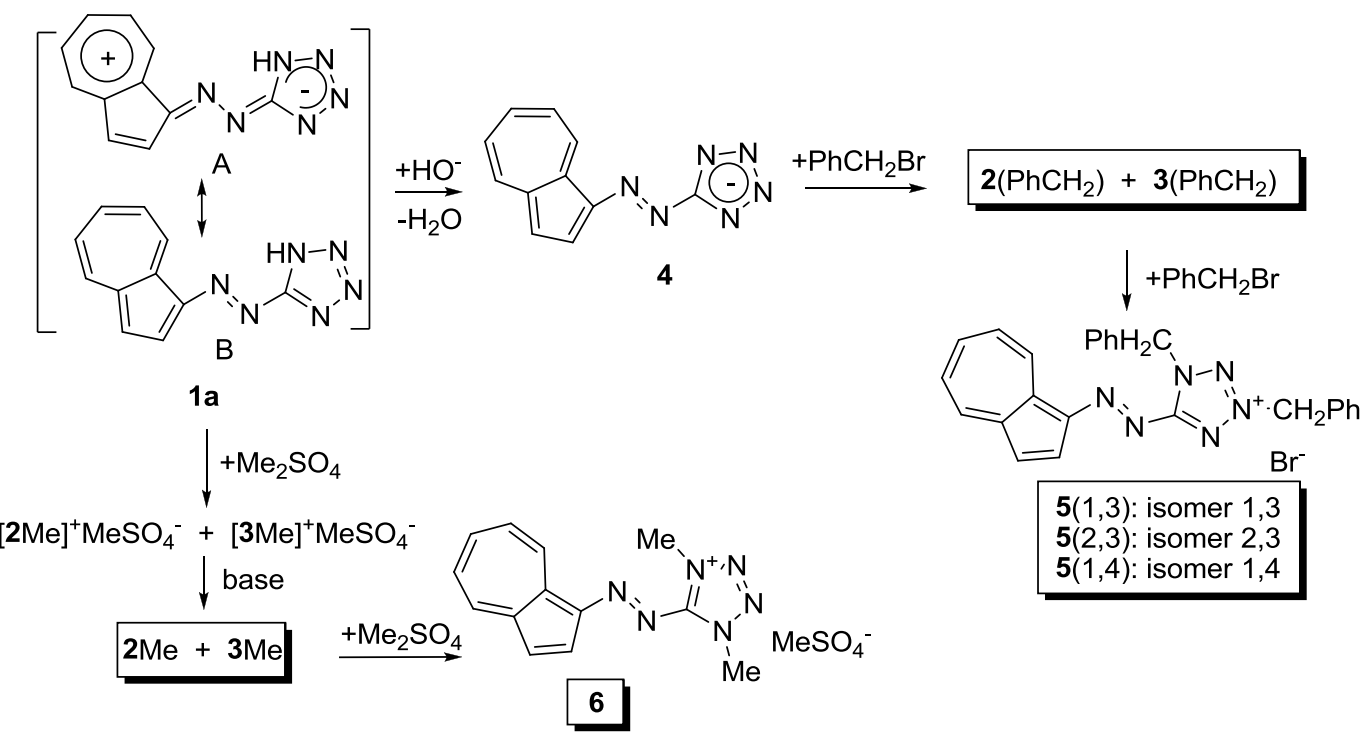

Scheme 4. Dialkylation of 5-(azulen-1-yldiazenyl)tetrazole, 1a, in excess of alkylating agents

The methylation with methyl iodide failed, possible due to the low nucleophilicity of tetrazolate anions 4. However, dimethyl sulfate alkylates the diazene after a long reaction time (24 hours for $74 \%$ yield), at room temperature with a high excess of reagent and in the absence of base. Possible, here the reaction route involves the formation of cations $[2 \mathbf{M e}]^{+}$and $[3 \mathbf{M e}]^{+}$, stabilized by the involvement of azulen-1-yl moiety, which after proton elimination gives the products $\mathbf{2 M e}$ and $3 \mathbf{M e}$ (Scheme 4). From the NMR spectra, can be estimated an inversed ratio between the isomers toward the benzylated products. With higher excess of dimethyl sulfate, both symmetric positions 1 and 4 are methylated (compound 6). 


\section{Characterization and properties}

For the characterization of synthesized compounds, together with the elemental analysis, the recorded NMR, UV-vis and mass spectra were of decisive help; therefore, in the following we will discuss some of the most relevant aspects of the obtained spectra. Between the properties of the studied diazenes, the acido-basic behavior, the redox parameters and the reactivity towards metals were considered of greater importance and consequently attention was paid to these aspects.

\section{Mass and NMR spectra}

The recording of molecular ion by mass spectroscopy of obtained diazenes was important in their identification and ESI method was used in this aim. The protonated diazenes are split generating as major products the 1-azulenediazonium ions and tetrazoles. This analytical procedure was also precious because it allowed the detection of the compounds generated in small amounts by alkylation.

For the diazenes reported in this work without substituent at nitrogen atoms only the azulene NMR spectra are significant therefore the substituent position at alkylated tetrazole moiety requires special attention.

Usually, the structure achievement of 1- and 2-alkylated isomers for 5-substituted tetrazoles after alkylation raises difficulties because they are hard to separate. However, several syntheses of individual 1-isomers on other unequivocal routes ${ }^{7}$ allowed its characterization whereas the 2 -isomers can be obtained almost solely in some syntheses by the substitution of 5-substituted tetrazoles. Therefore, the structure of isomers was generally assigned by comparison between the properties of similar compounds and, in this aim, NMR spectra played an important role. We try to apply this procedure to determine structure of methylated and benzylated products of here studied tetrazole diazenes.

Several papers presented the chemical shifts of N-methylated 5-substituted tetrazoles and, as can be seen from the Table 1. In all examples, proton chemical shifts of the methyl groups are higher for the isomer 2-Me than those reported for the isomer 1-Me [33]. Similar behavior was signaled for 1,1'- and 2,2'-dimethyl azotetrazole where $\delta$ is $4.38 \mathrm{ppm}$ for isomer 1,1'and $4.55 \mathrm{ppm}$ for the latter (in DMSO) [21].

Table 1.chemical shifts of protons of 1- and 2- methylated 5-substituted tetrazoles (in $\left.\mathrm{CdCl}_{3}\right)$

\begin{tabular}{|c|cccccccccc|}
\hline $\begin{array}{c}\text { Substituent at } \\
\text { nitrogen }\end{array}$ & $\mathrm{9}$ & $\mathrm{Me}$ & $t \mathrm{Bu}$ & $\mathrm{Ph}$ & $\mathrm{NH}_{2}$ & $\mathrm{Cl}$ & $\mathrm{CF}_{3}$ & $\mathrm{SO}_{2} \mathrm{Me}$ & $\mathrm{CO}_{2} \mathrm{Et}$ & $\mathrm{NO}_{2}$ \\
\cline { 2 - 32 } & $\mathrm{H}$ & 4.18 & 4.01 & 4.14 & 4.16 & 3.70 & 4.12 & 4.26 & 4.30 & 4.40 \\
\hline $1-\mathrm{Me}$ & 4.36 & 4.28 & 4.27 & 4.35 & 4.06 & 4.42 & 4.44 & 4.45 & 4.52 \\
\hline
\end{tabular}

When methylation is forced by the use of excess reagent and more severe reaction conditions, it continues with the generation of bis methylated tetrazoles, as salts [34,35]. For example [36], 1,3- and 1,4-dimethyl-5-vinyltetrazolium salts resulted at the methylation of 5-vinyltetrazole or of 1-methyl- or 2-methyl-5-vinyltetrazole with dimethyl sulfate. The $\delta$ belonging to salts show the deshielding of these values obviously due to the neighboring positive charge.

Table 2. ${ }^{1} \mathrm{H}$-chemical shifts of substituted 5-vinyltetrazoles and 5-(azulen-1-yldiazenyl) tetrazoles $(\delta$ in PPM)

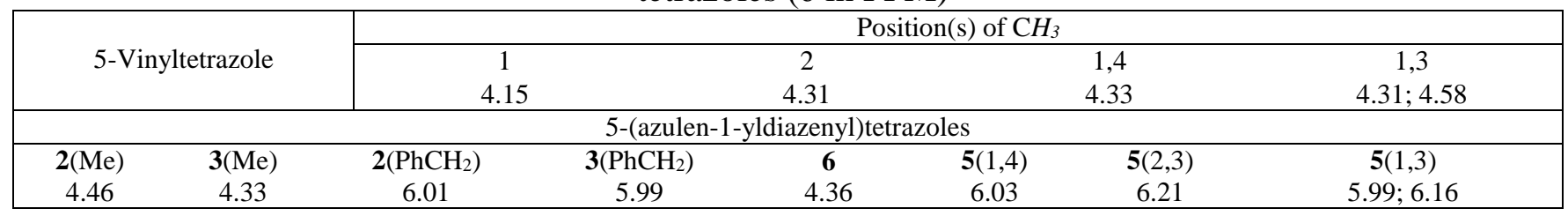

The data for chemical shifts of protons belonging to methyl or benzyl substituted at tetrazole in the studied diazenes, presented in Table 2, show a behavior similar to that described for the compounds 
analyzed above: the methyl group in position 2 is deshielded compared to that in position 1 . The same values for the protons of substituents at symmetrical positions 1,4 or 2,3 attest the uniform repartition of charge in the salts $\mathbf{5}(1,4), \mathbf{5}(2,3)$ and $\mathbf{6}$. The deshielding of the methyl groups in $\mathbf{6}$ compared cu $\mathbf{3}(\mathrm{Me})$ is low $(0.03 \mathrm{ppm})$ in spite of the positive charge of the tetrazolium system. This could be the result of the charge delocalization on the whole molecule, especially on the tropylium moiety of the azulene system. In the salt $\mathbf{5}(1,4)$, the $\mathrm{CH}_{2}$ groups also are little deshielded than in $\mathbf{3}\left(\mathrm{PhCH}_{2}\right)$. Less clear is why $2\left(\mathrm{PhCH}_{2}\right)$ has such a low value (it would be expected $\approx 6.15 \mathrm{ppm}$ ).

For azulene protons the comparison between chemical shifts of 5-(azulen-1-yldiazenyl)tetrazoles and (azulen-1-yldiazenyl)benzene, 7, or 2-(azulen-1-yldiazenyl)-1,3-thiazole, 8 [37], reveals the difference in the charge on the whole molecule.
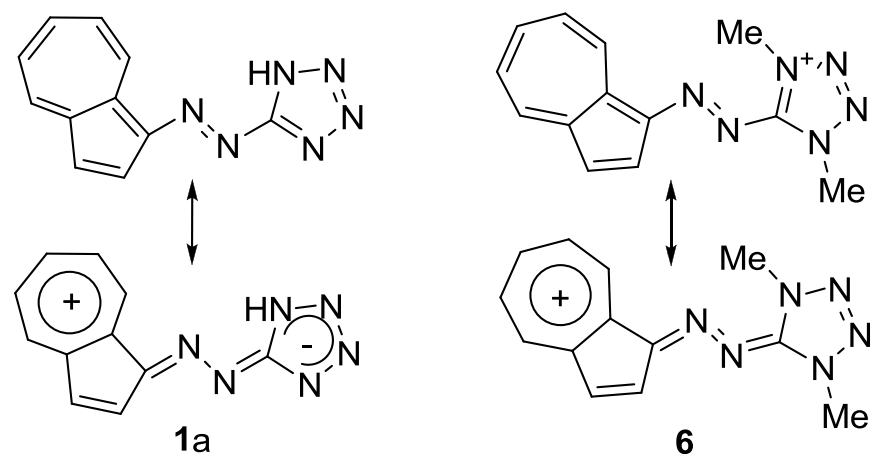

Scheme 5. Charge delocalization for 5-(azulen-1-yldiazenyl)tetrazole and its dimethylated derivative

As mentioned above, the neighboring positive charge produces the deshielding of neighboring protons. The $\delta$ values for protons $\mathrm{H}-4 \ldots \mathrm{H}-8$ of diazene 1a are higher than those of the same protons for compounds 7 or $\mathbf{8}$ (Table 3). The inductive effect of methyl in 2Me and in $3 \mathrm{Me}$ disturbs in some extent the zwitterion structure therefore the protons of seven-membered ring are shielded for these compounds towards those of the compound 1a. More pronounced is the deshielding of the protons $\mathrm{H}$ $4 \ldots \mathrm{H}-8$ for the positive charged salt $\mathbf{6}$. The slight decrease of $\delta$ for $\mathrm{H}-8$ when passing from compound $2 \mathrm{Me}$ to $2 \mathrm{Bz}$ (from 9.45 to $9.31 \mathrm{ppm}$ ) can be caused by the proximity of magnetic field generated by phenyl.

Table 3. Chemical shifts, $\delta$, for azulene protons of heteroaryl azulen-1-yldiazenes in acetone (in PPM)

\begin{tabular}{|cccccc|}
\hline Comp. & H-4 & H-5 & H-6 & H-7 & H-8 \\
\hline $\mathbf{7}$ & 8.38 & 7.37 & 7.80 & 7.45 & 9.36 \\
$\mathbf{8}$ & 8.31 & 7.40 & 7.78 & 7.52 & 9.19 \\
$\mathbf{1 a}$ & 8.71 & 7.76 & 8.14 & 7.87 & 9.44 \\
$\mathbf{2 M e}$ & 8.46 & 7.61 & 7.95 & 7.72 & 9.45 \\
$\mathbf{3 M e}$ & 8.46 & 7.52 & 7.87 & 7.57 & 9.45 \\
$\mathbf{6}$ & 9.00 & 8.36 & 8.57 & 8.43 & 9.41 \\
\hline
\end{tabular}

\section{UV-Vis spectra}

During our research on (azulen-1-yl)diazene containing five-membered heterocycles their UV-vis spectra were thoroughly investigated and several recorded absorption maxima are shown in Table 4. While the most blue-shifted absorption maxima are observed for the least polarizable heterocycles or phenyl, the highest absorption maxima is present for thiazole. 2-Azothiazoles [38] are the best chromophores among the azoheterocycles inducing high bathochromic effects and azulen-1-yl moiety greatly potentiate their properties. The absorption maximum in visible of 5-(azulen-1-yldiazenyl) tetrazole takes an intermediate position between these two limits. 
Table 4. Absorption maxima of some (azulen-1-yldiazenyl)aryls $\left(\lambda_{\max }\right.$ (in $\left.\mathrm{nm}\right)$ )

\begin{tabular}{|ccccc|}
\hline Aryl & Phenyl (7) & Oxadiazole $^{\text {a }}$ & Tetrazole (1a) & Thiazole (8) \\
\hline$\lambda_{\max }$ & 422 & 429 & 433 & 475 \\
\hline
\end{tabular}

As mentioned above, 5-(azulen-1-yldiazenyl)tetrazoles are soluble in water both in strong acidic and in basic media, however are slightly soluble in mild acidic medium. This can be explained by the formation of soluble tetrazolium salts in basic medium and tetrazole protonated salts generation in the strong acid presence. The mild acidic medium provides the isoelectric point at which the compounds possess compensated charges. The conjugation being at its maximum value, here are observed the absorption maxima in visible region (Table 5).

A bathochromic shift is recorded at nitrogen methylation from $433 \mathrm{~nm}$ for 1 a to $448 \mathrm{~nm}$ for $\mathbf{3 M e}$.

Table 5. Acido-basic behavior of unsubstituted azulenyldiazenyl-aryls in water ( $\lambda_{\max }$ in $\mathrm{nm}$ )

\begin{tabular}{|cccc|}
\hline Compound & $\begin{array}{c}\text { Basic-neutral } \\
\text { (tetrazolium salt) }\end{array}$ & $\begin{array}{c}\text { Mild acid } \\
\text { (zwitterionic structure) }\end{array}$ & $\begin{array}{c}\text { Strong acid } \\
\text { (protonated tetrazole) }\end{array}$ \\
\hline 1a & 448 & 468 & 443 \\
1b & 436 & 475 & 431 \\
1c & 441 & 468 & 431 \\
1d & 480 & 485 & 449 \\
1e & 471 & 475 & 431 \\
\hline
\end{tabular}

The two tautomers of 5-(azulen-1-yldiazenyl)tetrazoles unsubstituted at tetrazole ring present quite different dipole moments. Therefore, it is expected that the equilibrium between these tautomeric forms to vary in function of the solvent polarity with the absorption maxima higher in polar solvents. As resulted from Table 6 this assumption is only to some extent correct. For example, the solvents with acidic properties (traces of $\mathrm{HCl}$ in $\mathrm{CHCl}_{3}$ ) induce a bathochromic shift whereas those with basic properties (dioxane), have a hypsochromic effect.

Table 6. Solvatochromy of 5-(azulen-1-yldiazenyl)tetrazole, 1A

\begin{tabular}{|llllllll|}
\hline Solvent & dioxane & $\mathrm{CHCl}_{3}$ & $\mathrm{THF}$ & $\mathrm{Me}_{2} \mathrm{CO}$ & $\mathrm{MeNO}_{2}$ & $\mathrm{DMSO}$ & $\mathrm{H}_{2} \mathrm{O}$ \\
\hline$\varepsilon$ & 2.25 & 4.81 & 7.58 & 20.7 & 35.87 & 46.7 & 80.1 \\
$\lambda_{\max }(\mathrm{nm})$ & 434 & 455 & 452 & 441 & 458 & 446 & 447 \\
\hline
\end{tabular}

\section{Acido-basic behavior of 5-(azulen-1-yldiazenyl)tetrazoles}

Comprehensive reviewed data on the acido-basic properties of tetrazole derivatives ${ }^{4}$ underlines the difficulty encountered in these studies due to the presence of two tautomers for 5-substituted tetrazoles with different acidities (Table 7). Usually, the acidity of the 5-substituted tetrazoles was estimated from the degree of dissociation of 1-H tautomer, considered predominant in solutions, using conductometric or potentiometric titration or UV-spectroscopy. Due to the similar size, spatial arrangement of the nitrogen lone pairs and molecular electrostatic potential, 5-substituted tetrazoles, can be considered isosteres with carboxylic group, having a similar behavior as weak acids with $\mathrm{pK}_{\mathrm{a}}$ 4.5-4.9 [7]. The substituents at C5 influence their acidity: while the EWGs increase the acidic strength those EDGs decrease this property (Table 7).

Table 7. Acidity constants (in water) and basicity constants (in aqueous $\mathrm{h}_{2} \mathrm{SO}_{4}$ ) for several tetrazoles [4] and 5-(azulen-1-yldiazenyl)tetrazoles (in water)

\begin{tabular}{|c|c|c|c|c|c|c|c|c|c|c|}
\hline & \multicolumn{6}{|c|}{ tetrazoles substituted in position 5} & \multicolumn{4}{|c|}{ 5-(azulen-1-yldiazenyl)tetrazoles } \\
\hline & $\mathrm{H}$ & $\mathrm{Me}$ & $\mathrm{Ph}$ & $\mathrm{Cl}$ & $\mathrm{Br}$ & $\mathrm{NH}_{2}$ & $1 \mathbf{a}$ & 1b & 1c & 1e \\
\hline $\mathrm{pK}_{\mathrm{a}}$ & $\begin{array}{c}4.86 / \\
4.70\end{array}$ & $\begin{array}{l}5.56 / \\
5.50\end{array}$ & 4.83 & 2.07 & 2.13 & 6.0 & 3.78 & 3.45 & 3.41 & 4.60 \\
\hline $\mathrm{p} K_{\mathrm{BH}+}$ & -2.68 & -1.83 & -2.28 & - & -5.20 & - & 1.23 & - & - & - \\
\hline
\end{tabular}


As results from Table 7, the acidity of studied diazenyl tetrazoles, compared with that of other tetrazoles substituted at position 5, is higher than that for tetrazole with $\mathrm{R}=\mathrm{H}, \mathrm{Me}, \mathrm{Ph}, \mathrm{NH}_{2}$ and lower than for tetrazole with $\mathrm{R}=\mathrm{Br}, \mathrm{Cl}$. The opposite effect was observed on the basicity.

\section{Interaction with metals}

The metal atom can be integrated in metal derivatives of tetrazole by linking it to heterocycle by covalent, ionic or coordinative bond depending on the preparation procedures, nature of the metal, tetrazole structure as well as on the synthesis conditions. As a result of this wide diversity, more than 300 articles have been published in this field [40,41]. Good prospects for the practical use of such derivatives have fully encouraged their study. For example, very recently, the transition metal complexes with bidentate ligand 2-(1H-tetrazol-5-yl)pyridine was prepared and used for photonic applications [42]. The researches directed to the behavior of 5,5'-azotetrazoles in the presence of alkaline earth ions have been started since 1898 [13]. In fact, it is known that the parent diazene is unstable at room temperature if it is not preserved as its salts. To obtain the salts with different metallic ions, the initially generated sodium [43] or barium [44] salts were treated with different metallic salts, in order to precipitate the less soluble ligand salts.

The complexes generated from 5-substituted tetrazoles can contain tetrazole anions or neutral heterocycle. While the first are obtained usually in basic media, the last are obtained in neutral or acidic media [41].

When the solution of 5-(azulen-1-yldiazenyl)tetrazole (1a) in DCM, was stirred with an equivalent of silver nitrate (solid) and one equivalent of sodium hydroxide (1N solution) precipitated the low soluble silver salt (Scheme 6). Unfortunately, the salt cannot be highlighted as such by mass spectroscopy, ESI experiment, due to its complete ionization. However, the signal of corresponding anion $(M=223)$ was found by the negative ESI experiment while the silver ions are present in the positive experiment. Further, at the excess of 1a and hydroxide, the obtained salt forms a complex with another salt anion, which was registered on the negative ESI experiment with $\mathrm{M}=553 / 555$ (Scheme $6)$.
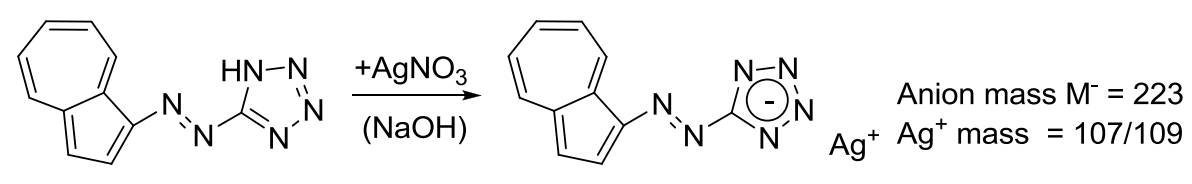

1a

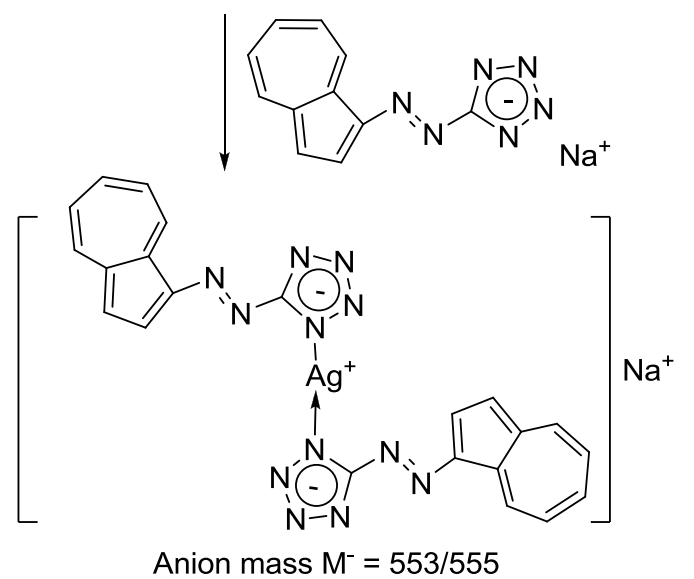

Scheme 6. Interaction of 5-(azulen-1-yldiazenyl)tetrazole anion with silver nitrate

It was interesting to note that the ligand chromophore 1a interact with the metal ions, such as iron (II) or (III) producing a color variation and leading to an almost linear variation between the maxima 
of absorption, molar extinction and the concentration of metal in solution (Fig.1). These correlations could be used for the dosation of such metal ions.

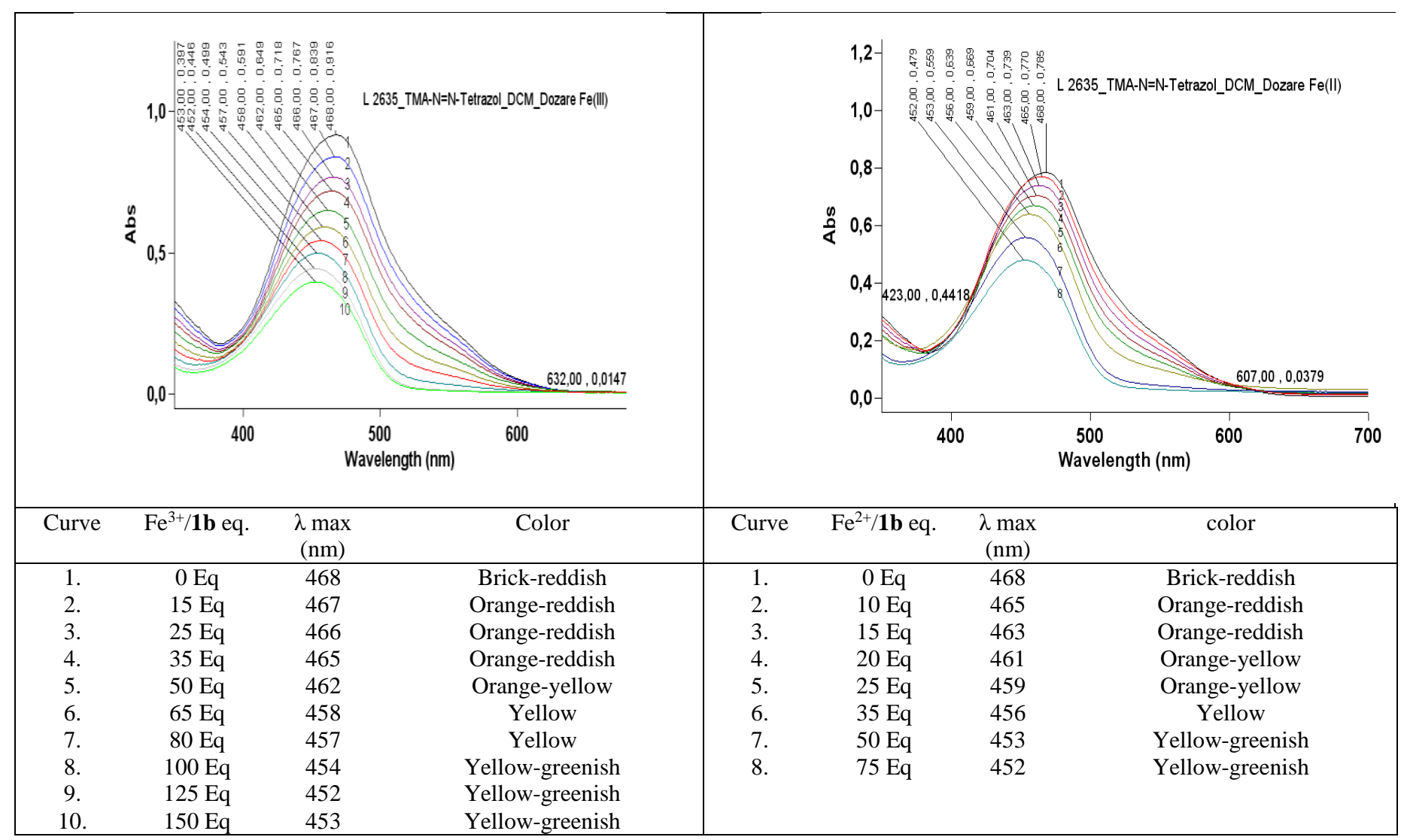

Figure 1. Variation between the maxima of absorption of $\mathbf{1 b}$, molar extinction and the amount (concentration) of the iron (II) or (III) present in solution

\section{Electrochemical data}

It seems that data on the redox behavior of tetrazole derivatives have not yet been reported in the literature with the exception of the summary study on cathodic behavior of several tetrazole derivatives $\left(\mathrm{E}_{1 / 2}\right.$ for tetrazole, $-1.45 \mathrm{~V}$ and for 5-methyltetrazole, $-1.60 \mathrm{~V}$ ) [45]. Therefore, we considered interesting to investigate the electrochemical properties of synthesized 5-(azulen-1-yldiazenyl) tetrazoles and the preliminary data will be described below [46]. It also seems useful to compare the results with those for other related azulenic diazenes (Scheme 1) [46]. The azulen-1-yl moiety is the most sensitive part of azulenyl diazenes against oxidation. However, was signaled the increase in oxidation potentials and decrease in reduction potentials with the electronegativity of the second substituent at azo bond. The highest stability of phenyl results in the highest oxidation potential of azobenzene (Table 8). The next downward value $(0.914 \mathrm{~V})$ was found for the parent 5-(azulen-1yldiazenyl)tetrazole, 1a, due to the strongest electron withdrawing effect of tetrazole among all heterocycles we have considered. Replacement of the 5-tetrazolyl moiety in 1a with 2-thiazolyl, 8 [47], or 2-(1,3,4-thiadiazolyl) [48] decreases the oxidation potential with 0.313 and $0.099 \mathrm{~V}$, respectively (Table 8$)$.

Replacement of phenyl moiety in (azulen-1-yldiazenyl)benzene, 7, with 5-tetrazolyl (1a) decreases the reduction potential by $0.095 \mathrm{~V}$ whereas another azulen-1-yl diazenes containing heterocycles are reduced easier.

As expected, the presence of alkyl substituents on tetrazole moiety with their electron-releasing effect promotes the electron elimination favoring oxidation and preventing reduction. The observed complex redox behavior of 5-(azulen-1-yldiazenyl)tetrazoles has suggested us to continue the study of electrochemical behavior of these compound classes and research is in progress. 
Table 8. Oxidation and reduction potentials

\begin{tabular}{|c|ccccccccc|}
\hline Potential & \multicolumn{8}{c|}{ Compound } \\
\cline { 2 - 10 }$(\mathrm{V})$ & $\mathbf{1 a}$ & $\mathbf{1 b}$ & $\mathbf{1 d}$ & $\mathbf{1 e}$ & $\mathbf{1 c}$ & $\mathbf{7}$ & $\mathbf{8}$ & $\mathrm{Tdaz}^{\mathrm{a}}$ & $\mathrm{Ph}-\mathrm{N}_{2}-\mathrm{Ph}$ \\
\hline $\mathrm{E}_{\mathrm{ox}}$ & 0.914 & 0.703 & 0.672 & 0.603 & 0.540 & 0.620 & 0.601 & 0.815 & 1.320 \\
$\mathrm{E}_{\text {red }}$ & -1.768 & -1.833 & -1.889 & -1.951 & -1.910 & -1.673 & -1.406 & -1.207 & -1.733 \\
\hline
\end{tabular}

aTdaz: 2-(Azulen-1-yldiazenyl)-1,3,4-thiadiazole [48]

\section{Conclusions}

5-(azulen-1-yldiazenyl)tetrazoles were prepared in good yields starting from tetrazole 5-diazonium salts, which was obtained by 5 -aminotetrazole diazotization in strong acid solution, followed by coupling with azulenes in the presence of pyridine. The tetrazole moiety was monoalkylated in 1- and in 2- position using dimethyl sulfate for methylation and benzyl bromide for benzylation. The excess of alkylating agent generated in small amount tetrazole dialkylated salts. The NMR and UV-vis spectra were compared with those of other azulene diazenes. Attention was paid to the acido-basic behavior of 5-(azulen-1-yldiazenyl)tetrazoles and to the interaction of compound 1a with metals. Oxidation and reduction potentials of prepared diazenes were recorded using differential pulse method (DPV).

\section{References}

1.BLADIN, J.A., Chem. Ber., 18, 1885, p. 1544

2.BUTLER, R.N., Adv. Heterocyl. Chem., 21, 1977, p. 323

3.WITTENBERGER S.J., Prep. Proc. Int.: New J. Org. Synth., 26, 1994, p. 499

4.OSTROVSKII, V.A., KOLDOBSKII, G I., TRIFONOV R.E. in Comprehensive Heterocyclic Chemistry III, Oxford, Elsevier, 2008, Vol. 6., Tetrazoles, 257-423.

5. SARVARY, A., MALEKI A., Molecular Diversity, 19, 2014, p. 189

6. SINGH, R.P., VERMA, R.D., MESHRI, D.T., SHREEVE J.M., Angew. Chem. Int. Ed. 45, 2006, p. 3584

7. NEOCHORITIS, C.G., ZHAO, T., DOMLING A., Chem. Rev. 119, 2019, p. 1970

8. OSTROVSKII, V.A., KOREN A.O., Heterocycles, 53, 2000, p. 1421

9. ROH, J., VAVROVA, K., HRABALEK A., Eur. J. Org. Chem., 2012, p. 6101

10. AROMIA, G., BARRIOSA, L.A., ROUBEAUB, O., GAMEZ, P., Coord. Chem. Rev., 225, 2011, p. 485

11. POPOVA, E.A., TRIFONOV, R.E., OSTROVSKII, V.A., ARKIVOC, 2012 (i) p. 45

12. KLAPÖTKE, T.M., STEIN, M., STIERSTORFER J., Z. Anorg. Allg. Chem. 634, 2008, p. 1711

13. THIELE, J., Justus Liebigs Ann. Chem., 303, 1898, p. 57

14. HAMMERL, A., HOLL, G., KLAPOTKE, T.M., MAYER, P., NOTH, H., PIOTROWSKI, H., WARCHHOLD, M., Eur. J. Inorg. Chem., 2002, p. 8342

15.HAMMERL, A., HISKEY, M.A., HOLL, G., KLAPOTKE, T.M., POLBORN, K., STIERSTORFER, J., WEIGAND, J., Chem. Mater., 17, 2005, p. 3784

16. STEINHAUSER, G., GIESTER, G., WAGNER, C., LEOPOLD, N., STERBA, J.H., LENDL, B.,BICHLER, M., Helv. Chim. Acta, 92, 2009, p. 1371

17. STEINHAUSER,G., GIESTER, G., LEOPOLD, N., WAGNER, C., VILlA, M., Helv. Chim. Acta, 92, 2009, p. 2038

18. STEINHAUSER, G., GIESTER, G., LEOPOLD, N., WAGNER, C., VILLA, M., MUSILEK, A., Helv. Chim. Acta, 93, 2010, p. 183

19.THIELE, J., Justus Liebigs Ann. Chem., 270, 1892, p. 54

20.THOTTEMPUDI, V., GAO, H., SHREEVE, J.M., J. Am. Chem. Soc., 133, 2011, p. 6464

21.SEREBRYANSKAYA, T.V., MATULIS, V.E., LYAKHOV, A.S., VOITEKHOVICH, S.V., GAPONIK, P.N., IVASHKEVICH O.A., Heteroatom Chem. 21, 2010, p. 24

22.KLAPOTKE, T.M., PIERCEY, D.G., Inorg. Chem.50, 2011, p. 2732

23.SHEGAL, I.L., STANOVKINA, K.V., KOVALENKO, N.G., SHEGAL, L.M., Chem. Heterocycl. Compd., 1974, p. 369 
24. QIU, Y.-X., LIN, W.-N., LI, Z.-X., YANG, Z.-J. O., YANG, L., DONG, W., J. Coord. Chem., 68, 2015 , p. 3945

25. GOERDELER, J., HAUBRICH, H., Chem. Ber. 93, 1960, p. 397

26. ANDERSON, JR. A.G., TAZUMA J.J., J. Am. Chem. Soc., 75, 1953, p. 4980

27. RAZUS A. C., BIRZAN, L., ARKIVOK, 2018(iv), p. 1

28. PRATSINIS, H., HAROUTOUNIAN, S.A., Nat. Prod. Lett., 16, 2002, p. 201

29. BUTLER, R. N., Chem. Rev., 75, 1975, p. 241

30. EFIMOVA, YU.A., ARTAMONOVA, T.V., KOLDOBSKII, G.I., RUSS. J. Org. Chem., 45, 2009, p. 725

31. EFIMOVA, J.A., MASHKOVA, E. A., ARTAMONOVA, T. V., KOLDOBSKII, G. I., Chem. Heterocycl. Comp. 44, 2008, p. 498

32. SPEAR, R.J., Aust. J. Chem. 37, 1984, p. 2453

33. QUAST, H., BIEBER, L., MEICHSNER, G., Liebiegs Ann. Chem., 1987, 469

34. VOITEKHOVICH, S.V., GAPONIK, P.N., IVASHKEVICH, O.A., Russ. Chem. Rev. 71, 2002, p. 721

35. GAPONIK, P.N., IVASHKEVISH, O.A., NAUMENKO, V. N., KOVALYOVA, T. B., T. N. ANDREEVA, A. O. KOREN Spectrochim. Acta. 49A(1), 1993, p. 135

36. RAZUS, A.C., BIRZAN, L., NAE, S., SURUGIU, N., CIMPEANU, V., J. Heterocycl. Chem., 40, 2003, p. 995

37. DIKEY, J.B., TOWNE, E.B., BLOOM, M.S., MOORE, W.H., HILL, H.M., HEYNEMANN, H., HEDBERG, D.G., SIEVERS, D.C., OTIS, M.V., J. Org. Chem., 24, 1959, p. 187

38. RAZUS, A.C., BIRZAN, L., CRISTEA, M., TECUCEANU, V., DRAGHICI C., Rev. Chim.(Buc) 66, 2015, p. 1074

39. GAPONIK, P.N., VOITEKHOVICH, S.V., IVASHKEVICH, O.A., Russ. Chem. Rev. 75, 2006, p. 507

40. KLAPOTKE, T. M., STEIN, M., STIERSTORFER, J. Z. Anorg. Allg. Chem. 634, 2008, p. 1711

41. POPOVA, E.A., TRIFONOV, R.E., OSTROVSKII V.A. ARKIVOC 2012 (i) p. 45

42. MALIK, R.A., BHAT, N.G., YADAV, R,. SINGH, N., KUMAR, G., MAL S., Asian J. Chem. 30, 2018, p. 520

43. HAMMERL, A., HOLL, G., KLAPÖTKE, T. M., MAYER, P., NÖTH, H., PIOTROWSKI, H., WARCHHOLD M., Eur. J. Inorg. Chem., 2002, p. 8342

44. HAMMERL, A., HOLL, G., KLAPOTKE, T. M., MAYER, P., NÖTH, H., PIOTROWSKI, H., WARCHHOLD M., Eur. J. Inorg. Chem., 2002, p. 8342

45. NIYAZYMBETOV, M.E., PETROSYAN, V.A., KOZLOV, V.V., PEVZNER M.S., $J$. Electroanal. Chem., 338, 1992, p. 239

46. ***Some electroactive properties of diazenyl 1a for modified electrodes have been presented as a poster in Croatia. E. Diacu, E.-M. Ungureanu, A. A. Ivanov, S. Pistol, V. Anastasoaie, C. Omocea, L. Birzan.The 7 th Regional Symposium on Electrochemistry - South East Europe The Kurt Schwabe Symposium Split, Croatia, May 27-30, 2019 p RSE-SEE7 EAS-P-12.

47.UNGUREANU, E.-M., CRETU , M. -S., BUICA, G.-O., RAZUS, A. C., BIRZAN L., "Electrochim. Acta, 53(24), 2008, p. 7089

48. RAZUS, A. C., BIRZAN, L., CRISTEA, M., TECUCEANU, V., DRAGHICI, C., HANGANU, A., MAGANU, M., PINTILIE, L., UNGUREANU, E.-M., Rev. Chim., 70(5), 2019, p. 1518

$\overline{\text { Manuscript received: } 03.16 .2020}$ 